in their later years. To date there has been no study on the effect of AN and AAN on BMD in young Asian adolescents.

Objectives In this retrospective study, we compare BMD and baseline characteristics in Adolescents from Singapore with AN and AAN, to find any difference in BMD Z-score between $\mathrm{AN}$ and AAN, and any predictive factors for low bone density in adolescents with EDs.

Methods We reviewed electronic medical records for all patients below the age of 18, who were treated for Eating Disorders at KK Women's and Children's Hospital between 2010 and $2020(\mathrm{n}=507)$.

Results 318 AN and 141 AAN cases were identified within the study period. Mean age of all cases is $14.08 \pm 1.49$ years with mean duration of illness being $8.06 \pm 6.15$ months. Females made up $92.8 \%$ of AN cases, compared to $84.4 \%$ of AAN cases $\left(\chi^{2}(1)=7.739 ; p=0.005\right) . \% \mathrm{mBMI}$ at presentation was significantly lower for AN than AAN (74.55 \pm 7.27 versus $97.12 \pm 9.95, t(210)=-24.18, p<0.001) .29 \%$ of AAN patients were premorbidly overweight $(\max$ IBW $>120 \%)$, compared to $4.6 \%$ of AN patients $\left(\chi^{2}(1)=42.97 ; p<\right.$ 0.001). There were significantly higher BMD Z-scores in AAN compared to AN for both the spine $(1.55 \pm 1.63$ versus 0.29 $\pm 1.67, \mathrm{t}(179)=-4.22, \mathrm{p}<0.001)$ and femur $(0.48 \pm 0.93$ versus $0.23 \pm 1.19, \mathrm{t}(194)=-3.63, \mathrm{p}<0.001)$ region. In our linear regression model, BMD Z-score was not associated with duration or magnitude of weight loss as well as duration of amenorrhea or overweight status.

Conclusions From the findings of our study, we conclude that asian adolescents with AAN demonstrated higher BMD Zscores for both spine and femur compared to their AN counterparts. There was no predictive factors for low bone density. These findings may be instructive in informing guidelines for the management of Asian adolescents with AN and AAN.

\section{INTRAVENTRICULAR HAEMORRHAGE IN A UGANDAN COHORT OF LOW BIRTH WEIGHT NEONATES: THE IVHU STUDY}

Rachael MacLeod, Noella Okalany, Leigh E Dyet, Cornelia F Hagmann, Frances M Cowan, Kathy Burgoine. UK

\subsection{6/bmjpo-2021-RCPCH.210}

Background Globally, 15 million neonates are born prematurely every year, over half in low income countries (LICs). Premature and low birth weight neonates have a higher risk of intraventricular haemorrhage (IVH). There are minimal data regarding IVH in sub-Saharan Africa. This is one of the first studies of IVH in LBW neonates in a LIC in sub-Saharan Africa and the first in east Africa.

Objectives The objective of this study is to examine the incidence, severity and timing of and modifiable risk factors for IVH amongst low-birth-weight neonates in Uganda.

Methods This is a prospective cohort study of neonates with birthweights of $\leq 2000 \mathrm{~g}$ admitted to a neonatal unit (NU) in a regional referral hospital in eastern Uganda. Maternal data were collected from interviews and medical records. Neonates had cranial ultrasound (cUS) scans on the day of recruitment and days 3, 7 and 28 after birth. Risk factors were tabulated and are presented alongside odds ratios (ORs) and adjusted odds ratios (aORs) for IVH incidence. Outcomes included incidence, timing and severity of IVH and 28-day survival.
Results Overall, 120 neonates were recruited. IVH was reported in 34.2\% of neonates; $19.2 \%$ had low grade (Papile grades 1-2) and $15 \%$ had high grade (Papile grades $3-4$ ). Almost all IVH (90.2\%) occurred by day 7, including $88.9 \%$ of high grade IVH. Of those with known outcomes, $70.4 \%$ $(81 / 115)$ were alive on day 28 and survival was not associated with IVH.

We found that vaginal delivery, gestational age (GA) $<32$ weeks and resuscitation in the NU increased the odds of IVH. The aOR for having any IVH was 3.5 (95\% CI 1.0116.45), comparing vaginal delivery with Caesarean delivery. Compared with neonates of $\geq 32$ weeks GA, neonates of GA $<32$ weeks had increased odds of any IVH, aOR $6.70(95 \%$ CI 1.6-31.02), high grade IVH, aOR 8.18 (95\% CI 1.1869.37), and low grade IVH, aOR 6.70 (95\% CI 1.12-46.9). Neonates who required resuscitation in the $\mathrm{NU}$ also had increased odds of any IVH, aOR 5.10 (95\% CI 1.23-26.36) and high grade IVH aOR 9.24 (95\% CI 1.83-54.38). Neonates who were SGA (small for gestational age, <10th centile) had increased odds of low grade IVH, aOR 9.96 (95\% CI 1.83-71.84). Of the 6 neonates who received 2 doses of antenatal steroids, none had IVH.

Conclusions This study found that in this resource limited NU in a regional referral hospital in eastern Uganda, more than a third of neonates born weighing $\leq 2000 \mathrm{~g}$ had an IVH and majority of these occurred by day 7 . We found that vaginal delivery, GA < 32 weeks, resuscitation in the NU and being SGA were associated with increased odds of having an IVH. This study had a high rate of SGA neonates and the risk factors and relationship of these with IVH in this setting needs further investigation. The role of antenatal steroids in the prevention of IVH in LICs also needs urgent exploration.

\section{THE UTILITY OF C-REACTIVE PROTEIN FOR SCREENING AND DIAGNOSIS OF EARLY-ONSET INFECTION IN TERM NEONATES}

Xuelian Wang, Hugh Simon Lam. China

10.1136/bmjpo-2021-RCPCH.211

Background Early onset sepsis (EOS) remains a significant cause of neonatal mortality and morbidity. About $10 \%$ of term neonates were exposed to antibiotics for EOS risk, despite a very low incidence (around 1\%o) in this group especially after the introduction of intrapartum antibiotic prophylaxis. One of the main reasons for over treating the neonates is the lack of ideal diagnostic biomarkers. C-reactive protein (CRP) and complete blood count (CBC) are most commonly used biomarkers for EOS, but the diagnostic performance of CRP was poorly understood with most studies on late onset sepsis. In the past 15 years, our neonatal unit has used CRP systematically in neonates with risk or signs of EOS, providing a good opportunity to analyze its diagnostic accuracy.

Objectives In this study, we aimed to evaluate the performance of CRP for early onset infection diagnosis in symptomatic neonates and screening in asymptomatic high-risk neonates.

Methods This is a retrospective study done in a tertiary neonatal care center in Hong Kong. Term neonates born during the period of 01/01/2005-30/06/2018 with blood taken for CRP testing in the first 72 hours were included. Their CRP results were included into the analysis if blood were taken before antibiotic treatment. Subjects were divided into four 
subgroups according to their infectious status and having clinical signs or not. CRP values were compared between neonates infected and not infected. Receiver operating characteristic (ROC) curves were constructed separately in symptomatic and asymptomatic groups. The sensitivity, specificity, positive predict value, negative predict value, likelihood ratios and posttest probability were determined using different cut-off values. Results 24,344 infants with 28,830 CRP results were included. Early onset infection was confirmed in 75 (0.31\%) cases, 68 of whom had EOS and 7 had local infections (urinary tract or eyes). Discrimination improved after 8 hours of birth in asymptomatic neonates (the area under the ROC curve (AUC) was 0.49 at $<8$ hours and 0.79 at $\geq 8$ hours), and 24 hours of birth in symptomatic neonates (the AUC was 0.65 at $<24$ hours and 0.75 at $\geq 24$ hours). High CRP values were less informative in symptomatic neonates than asymptomatic neonates (eg. the likelihood ratio for CRP>10 mg/L was 2.2 in symptomatic and 5 in asymptomatic neonates). Using cut off value of $10 \mathrm{mg} / \mathrm{L}$ at $\geq 24$ hours, sensitivity was $72 \%$ in symptomatic neonates and $83 \%$ in asymptomatic neonates, and specificity was $69 \%$ and $86 \%$ respectively. Positive predict values were very low $(0.3-6 \%)$.

Conclusions Diagnostic performance of CRP was poor within 24 hours of birth. However, in asymptomatic neonates, high CRP in 8-24 hours was informative of infection. Because of the low positive predict value, attention should be paid to overtreatment when using CRP for decision making.

\section{CONTEMPORARY TRENDS IN GLOBAL MORTALITY OF NEONATAL SEPSIS: A SYSTEMATIC REVIEW AND META- ANALYSIS}

Wen Li Lee, Ming Ying Gan, Bei Jun Yap, Tammie Seethor, Bobby Tan, Shu-Ling Chong. Singapore and United States of America

\subsection{6/bmjpo-2021-RCPCH.212}

Background Sepsis causes death and morbidity in young infants. Globally, an estimated 1.3 - 3.9 million young infants experience sepsis and 400,000 - 700,000 die from sepsisrelated conditions annually. Even though there have been significant progress over the past twenty years in reducing young infant mortality, sepsis currently accounts for up to $15 \%$ of all young infant deaths. A thorough understanding of young infant sepsis can inform strategies that span prevention, diagnosis and intervention for young infant sepsis.

Objectives We aimed to perform a systematic review and meta-analysis to investigate the case fatality rates (CFRs) among young infants less than 90 days with sepsis globally.

Methods We used the Preferred Reporting Items for Systematic Reviews and Meta-analysis (PRISMA) 2009 guidelines. We searched PubMed, Cochrane Central, Embase and Web of Science for randomized clinical trials and observational studies in English language, published between 2010 to 2019. Studies involving young infants less than 90 days old with sepsis and reported CFRs were included. We obtained pooled CFRs estimates using the random effects model. Additional stratifications by gestation, birth weight, onset of sepsis (early onset was defined as $<72$ hours), source of sepsis and gross national income were also performed. Risk of bias was assessed using the Cochrane risk-of-bias tool for randomized controlled trials, and the Newcastle-Ottawa Scale for all observational studies.
Results Among 6314 articles screened, 240 studies with a total of 437,796 patients met the inclusion criteria and were included in our analysis. 99 came from high income countries, 44 from upper middle income countries, 82 from lower middle income countries, 6 from low income countries and 9 were conducted in multiple countries. Overall, the pooled CFR was 0.18 (95\% CI, 0.17-0.19). The CFR was the highest in low income countries $(0.25$ [95\% CI, 0.07-0.43]), followed by lower middle (0.24 [95\% CI, 0.21-0.26]), upper middle $(0.21$ [95\% CI, 0.18-0.24]) and lastly high income countries (0.12 [95\% CI, 0.11-0.13]).

Other factors associated with higher CFRs included prematurity $(0.23$ [95\% CI, 0.19-0.26] vs term CFR 0.10 [95\% CI, $0.08-0.13])$, low birth weight $(0.21$ [95\% CI, 0.19-0.24] vs normal birth weight 0.19 [95\% CI, 0.18-0.20]), early onset sepsis $(0.20$ [95\% CI, 0.17-0.24] vs combined (0.16 [95\% CI, $0.14-0.18])$ and hospital acquired infection (0.23 [95\% CI, $0.17-0.30]$ vs community acquired infections 0.21 [95\% CI, $0.10-0.33])$. Time trend analysis showed higher CFRs in the low income countries than the middle and high income countries. A decreasing trend in CFRs over time was observed in high and upper middle income countries, as compared to an increasing trend in lower middle and low income countries.

Conclusions While we saw a declining trend of young infant sepsis CFRs among high and upper middle income countries across the years, the increasing trend amongst lower middle and low income countries highlights a disparity in infant sepsis outcomes based on resource availability. We highlight specific vulnerable patient populations that should be further studied in order to reduce the global burden of young infant sepsis.

\section{MEDICATION ERRORS AND NEAR-MISSES IN A PEDIATRIC EMERGENCY DEPARTMENT: A RETROSPECTIVE REVIEW}

Ann Loh, Sung Shin Teng, Khai Pin Lee, Sashikumar Ganapathy. Singapore

\subsection{6/bmjpo-2021-RCPCH.213}

Background Medication errors (MEs) are a significant cause of preventable morbidity and mortality. The paediatric emergency department (ED) is a high-risk setting with high patient volume and acuity of care, serving a uniquely susceptible population where weight-based calculations render them vulnerable to dosing errors. Medications are also often kept in stock, and are not audited by a pharmacist prior to administration. Stress, noise, time pressures, and unfamiliarity with paediatric conditions amongst rotating trainees compound this risk.

Objectives To describe the occurrence and type of MEs in pediatric ED and to identify contributing factors.

Methods A retrospective review of all reported MEs in Singapore's largest tertiary pediatric ED from January 2013 to December 2019. MEs were reported via Risk Management System (RMS), while near-misses were extracted from RMS and the pharmacy department's Closed Loop Medication Management System. Descriptive statistics were used to present ME types, severity and contributing factors.

Results Of 101 MEs reported in RMS, 59\% were related to wrong dose, $22 \%$ to wrong medication and $6 \%$ to wrong patient. Wrong doses were related to duplicate dose $(48 \%)$, wrong weight (20\%), 10-fold errors (5\%) and calculation errors (5\%). Majority of MEs occurred during drug 